Fitt, A, Mphaka, M, Nagamine, A and Cuminato, J Goal sketching from a concise business case. A singular integro-differential equation model for dryout in LMFBR boiler tubes.

Fitt, A, Mphaka, M, Nagamine, A and Cuminato, J (2009) A singular integro-differential equation model for dryout in LMFBR boiler tubes. IMA Journal of Applied Mathematics, 75 (2). pp. 269-290.

Doi: 10.1093/imamat/hxp039

This version is available at https://radar.brookes.ac.uk/radar/items/35615212-3aa9-1e16-5ade-b161321fd25a/1/ Available on RADAR: October 2012

Copyright (C) and Moral Rights are retained by the author(s) and/ or other copyright owners. A copy can be downloaded for personal non-commercial research or study, without prior permission or charge. This item cannot be reproduced or quoted extensively from without first obtaining permission in writing from the copyright holder(s). The content must not be changed in any way or sold commercially in any format or medium without the formal permission of the copyright holders.

This document is the postprint version of the journal article. Some differences between the published version and this version may remain and you are advised to consult the published version if you wish to cite from it. 
IMA Journal of Applied Mathematics (2009) 1-22

doi: 10.1093/imamat/*****

\title{
A singular integro-differential equation model for dryout in LMFBR boiler tubes
}

\author{
J.A. Cuminato ${ }^{1}$, A.D. FitT ${ }^{2}$, M.J.S. MphaKa ${ }^{3} \&$ A. NAgAmine ${ }^{4}$ \\ ${ }^{1}$ Departamento de Matemática Aplicada e Estatística, Instituto de Ciências Matemáticas e \\ de Computacão, Universidade de São Paulo, P.O. Box 668 - 13560-970 - São Carlos - SP - \\ Brazil. \\ ${ }^{2}$ School of Mathematics, University of Southampton, Southampton, SO17 1BJ, UK. \\ ${ }^{3}$ Department of Mathematics and Computer Science, National University of Lesotho, P.O. \\ Roma 180, Maseru 100, Lesotho. \\ ${ }^{4}$ DCET, Universidade Estadual de Santa Cruz, Ilhéus, Brazil.
}

$$
\text { [Received on } * * * * * * * * \text { ] }
$$

\begin{abstract}
A two-dimensional steady model for the annular two-phase flow of water and steam in the steam generating boiler pipes of a Liquid Metal Fast Breeder Reactor (LMFBR) is proposed. The model is based on thin-layer lubrication theory and thin aerofoil theory. The exchange of mass between the vapour core and the liquid film due to evaporation of the liquid film is accounted for using some simple thermodynamics models, and the resultant change of phase is modelled by proposing a suitable Stefan problem. Appropriate boundary conditions for the flow are discussed. The resulting nonlinear singular integro-differential equation for the shape of the liquid film free surface is solved both asymptotically and numerically (using some regularisation techniques). Predictions for the length to the dryout point from the entry of the annular regime are made. The influence of both the traction $\tau$ provided by the fast flowing vapour core on the liquid layer and the mass transfer parameter $\eta$ on the dryout length is investigated.
\end{abstract}

Keywords: Singular Integral Equation, Dryout point, LMFBR

\section{Introduction}

In modern nuclear power plants, the raw material of nuclear energy production is natural uranium. This is principally because its two isotopes $U^{235}$ and $U^{238}$ possess nuclear characteristics that are very favourable to the production of atomic energy in a reactor. (See, for example, (Murray, 1996).) A typical nuclear reactor possesses two key components, namely a reactor/core component (or a fuel element) and a boiling/heat exchange component. Nuclear fission and energy conversion take place in the reactor component, where heat is generated and transferred to a coolant. Heat is then transferred from the coolant to water in the boiling component where steam is produced to drive turbines that generate electricity.

Nuclear reactors of many different types exist (see, for example (Collier \& Hewitt, 1987)). One factor that differentiates between various reactor designs is the type of coolant used. In a Liquid Metal Fast Breeder Reactor (LMFBR), for example, a liquid metal is used as the coolant. Liquid sodium is a common choice of coolant since it has a high boiling point of $883^{\circ} \mathrm{C}$ at atmospheric pressure, allowing the nuclear reactor to operate virtually unpressurised. Thus, in the event of a Loss Of Coolant Accident (LOCA) in which the integrity of system components is compromised, (see (Collier \& Hewitt, 1987)), the damage and spread of contaminated material is minimised. (The melting point of sodium is $98^{\circ} \mathrm{C}$; 


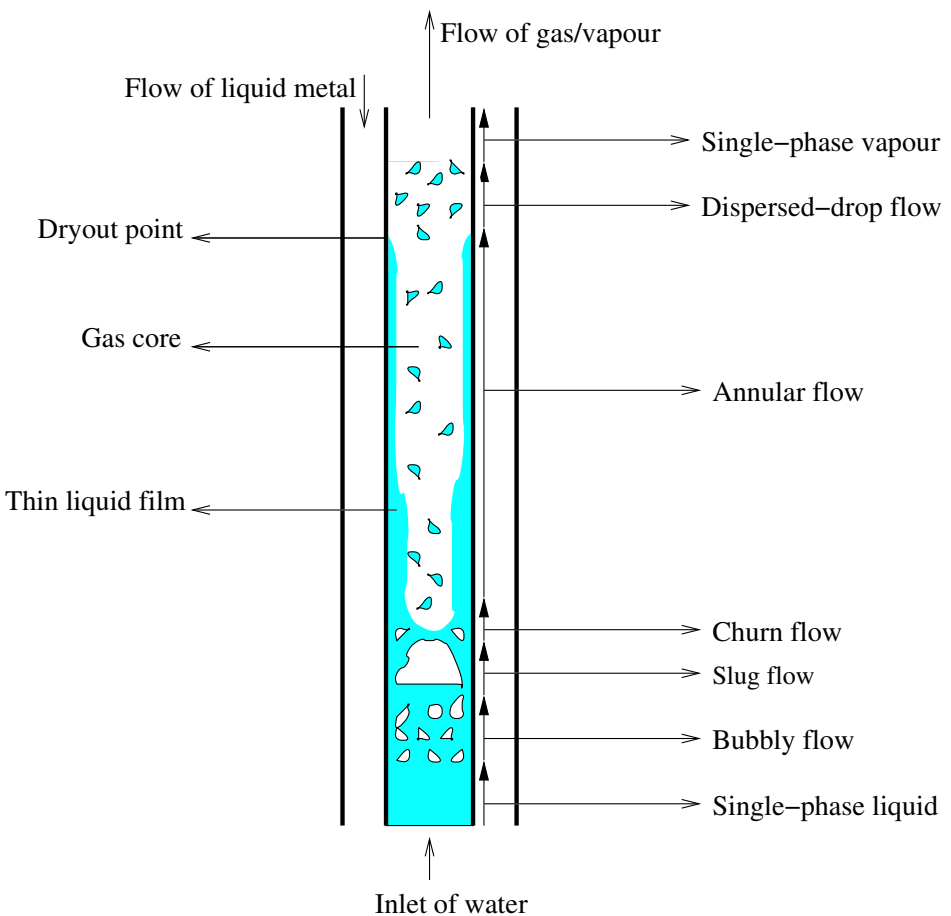

FIG. 1. A schematic representation of a vertical boiler tube.

all sodium pipes therefore have to be heated to avoid solidification at room temperature.)

The first fully functioning LMFBR was the EBR-1 (Experimental Breeder Reactor) at Idaho Falls, USA, which first generated electricity in 1951. Its successor EBR-2 successfully generated power (mainly for test and material proving purposes) continuously from 1967-1994. Both the French/European Superphenix and the Japanese MONJU LMFBRs were dogged by sodium leakage problems and neither is currently in operation, but the LMBFR principle has been triumphantly verified by the 560MW BN600 reactor in Beloyarskiy, Russia, which has operated commercially since 1981 and was still successfully producing power in 2008. For a much fuller discussion of the history of LMFBRs see, for example, (Murray, 1996).

The boiling component of a nuclear reactor is composed of bundles of steam generating boiler pipes. Water is pumped through the pipes, and heat is supplied from the liquid metal which flows in a countercurrent direction in outer casings surrounding the pipes. As the water temperature increases, the water begins to vapourise, driving a two-phase flow of water and steam. In a typical boiler pipe, the water and the steam generated give rise to a variety of flow regimes: these are shown schematically in figure 1 .

Which particular flow regime pertains depends in a complicated fashion upon the amounts of each phase present, external effects such as orientation of the pipe, and the flow parameters such as pressure and heat flux. Detailed descriptions of the various flow regimes are given, for example, in (Collier \& Thome, 1994) and (Wallis, 1969). To summarise briefly, the flow typically consists of a single-phase subcooled region near to the water inlet. The water in this subcooled region is heated to the saturation 
temperature. At some point along the pipe beyond this region the water becomes superheated and bubbles start to form at nucleation sites on the pipe wall. As the bubbles grow, they detach from the wall and begin to form a bubbly flow region where the vapour phase is distributed as discrete bubbles in a continuous liquid phase. As more and more bubbles are produced, they first amalgamate to become larger bubbles and then to form a slug flow region where vapour may be present across the whole width of the pipe. Under certain flow conditions churn flow, where the vapour regions in the plug flow break up to form irregular and unstable bubbles, may also be observed. Eventually, however, annular two-phase is established.

In the annular region, a relatively slowly-moving continuous liquid film on the pipe wall surrounds a core of fast flowing gas. The vapour core invariably contains liquid droplets which may originate from undercutting of waves that are present on the film surface. No appreciable bubble nucleation takes place at the pipe wall (where the liquid is superheated), and the dominant gas production mechanism is evaporation of the thin liquid film at its free surface. Liquid droplets in the gas core subsequently evaporate beyond the annular flow region to form a dispersed-drop flow region. Here, the fluid film on the pipe wall has completely evaporated and no liquid is present. Finally, the remaining liquid droplets in the gas core evaporate until only a single-phase vapour region is present.

The phenomenology described above has been greatly simplified, and there may be many other intermediate flow regimes such as wispy-annular flow. The progression of regimes described above represents the simplest scenario that can sensibly be addressed (see (Whalley, 1987)). The description of intermediate regimes is frequently a somewhat subjective matter (see, for example, (Azbel, 1981)) and the literature is by no means unanimous. The presence of different flow regimes may also be influenced by pipe orientation if the water inlet speed is sufficiently small. For example, in horizontal boiler pipes the gas bubbles often migrate to the upper side of the pipe. For low inlet speeds a stratified flow region may also be present after the plug flow region.

In this study we shall consider only the annular flow regime, since this is the predominant regime present in LMFBR boiler tubes (as well as in evaporators, condensation operations, natural gas pipelines and steam generating systems; see (Wallis, 1969)). At normal reactor operating conditions (for example, pressures of around $200 \mathrm{bar}$ and hence a water saturation temperature of $T_{s}=365^{\circ} \mathrm{C}$ ) the flow pattern maps in (Bennett et al., 1965) suggest that annular flow is present in at least 80 to 90 percent of the two-phase flow region. The annular flow region is responsible for most of the water/steam mass transfer in the pipe, and terminates at the so-called "dryout point", where complete evaporation of the liquid film first occurs.

Careful control of the location of the dryout point is important if a reactor is to function properly. At the dryout point the pipe wall temperature increases sharply since the thermal conductivity of the gas phase, which is now in direct contact with the wall, is much less than that of the liquid phase. The determination of the position of the dryout point is not a trivial problem (see (Fisher \& Pearce, 1993)) since, for example, in the event that deposition of liquid drops occurs rapidly, the liquid film may reform, causing rewetting and a consequent drop in the temperature of the pipe wall. If the processes of dryout and rewetting occur periodically, thermal stresses may be set up in the wall which could lead to cracking of the pipe. A good understanding of dryout is therefore essential if one wishes to predict the lifetime of steam generating boiler pipes, Moreover the dryout process imposes a limit on the amount of evaporation that occurs in the pipes for a given value of the heat flux, and is of great importance in the design of evaporators, steam boilers and other types of nuclear reactors ((Bankhoff, 1994), (Collier $\&$ Thome, 1994)). Accordingly, we develop below a model to predict dryout point location.

The flow that we shall model falls into the general class of non-isothermal thin-film flows subject to an external air flow, and much previous work has been carried out on such flows. In particular, previous 
studies have considered evaporating thin films (Burelbach et al., 1988), the dryout of the microlayers surrounding a non-isothermal bubble (Wilson et al., 1999), wind-driven rain drops on a car windscreen (King \& Tuck, 1993), and non-evaporating drops in a shear flow (Dimitrakopoulos \& Higdon, 1997) and (Spelt, 2006), all of which are relevant to the model proposed below. Singular integral equationbased models for such flows tend to lead to challenging numerical difficulties: the range of numerical methods that may be required for various problems and the difficulties that might be present have also previkously been reviewed (Cuminato et al., 2007).

\section{Mathematical modelling}

Our basic premise will be to assume that the annular two-phase flow in the tube is steady and twodimensional, the flow in the thin liquid layer adjacent to the heated wall is governed by standard lubrication theory, the evaporation of the liquid proceeds according to a classical Stefan-type condition and the interaction between the fast-flowing gas core and the wall layer may be described using classical thin aerofoil theory. Parametric justification for these assumptions will be discussed below. The result of the model will be a nonlinear singular integro-differential equation (NSIDE) that will be studied using both asymptotic and numerical techniques.

\subsection{Liquid film region}

In the liquid film, the flow is governed by the Navier-Stokes equations

$$
\begin{gathered}
\underline{q}_{t}+(\underline{q} \cdot \nabla) \underline{q}=-\frac{1}{\rho} \nabla p+v \nabla^{2} \underline{q}, \\
\nabla . \underline{q}=0
\end{gathered}
$$

where $q=(u, v)^{T}$ denotes the fluid velocity, $t$ denotes time and $p, \rho$ and $v=\mu / \rho$ denote respectively the pressure, density and kinematic viscosity. For simplicity, we shall assume that both $\rho$ and $v$ are constant, and ignore the effects of gravity. If required, (for example, to study the effects of inclined reactor pipes) gravity may easily be incorporated into the pressure. To apply standard thin-layer theory, we nondimensionalise by setting $x=L \bar{x}, y=\varepsilon L \bar{y}, t=(L / U) \bar{t}, h=\varepsilon L \bar{h}, u=U \bar{u}, v=\varepsilon U \bar{v}$ and $p=\left(\mu U L / h_{0}^{2}\right) \bar{p}$, where an overbar denotes a non-dimensional variable, $x$ and $y$ denote distances along and normal to the pipe wall respectively, $L$ denotes the distance from the onset of annular flow to the dryout point, $h_{0}$ denotes the fluid film thickness at some known point in the annular flow regime (which will henceforth be designated $x=0), \mu$ denotes the fluid dynamic viscosity, $U$ denotes a typical flow speed in the film layer and $\varepsilon$ denotes the ratio $h_{0} / L$. Note that we do not know $L$; indeed, one of the primary purposes of this study is to determine it. We assume, however, that we know its order of magnitude. Using typical operating values from Appendix A, we find that the ratio of a typical liquid layer thickness $(\sim 0.5 \mathrm{~mm})$ to a typical tube radius $\sim 7 \mathrm{~mm})$ is small, thus justifying both a two-dimensional approach to the problem and the neglect of axisymmetric effects. The key non-dimensional parameters in the problem are therefore $\varepsilon$ and the reduced Reynolds number, which (using $L \sim 5 \mathrm{~m}$ ) are given respectively by

$$
\varepsilon=\frac{h_{0}}{L} \sim 10^{-4}, \quad \operatorname{Re} \varepsilon^{2}=\frac{L U \rho \varepsilon^{2}}{\mu} \sim 4 \times 10^{-3} .
$$

We therefore conclude that normal lubrication theory applies wherein there is a balance between viscous and pressure gradient forces in the flow direction. Omitting some standard calculations for purposes of 
brevity, we find that, in dimensionless variables,

$$
\begin{aligned}
\bar{u} & =\frac{1}{2} \bar{p}_{\bar{x}} \bar{y}(\bar{y}-2 \bar{h})+\bar{\tau} \bar{y} \\
\bar{v} & =\frac{1}{6} \bar{p}_{\bar{x} \bar{x}} \bar{y}^{2}(3 \bar{h}-\bar{y})+\frac{1}{2} \bar{p}_{\bar{x}} \bar{y}^{2} \bar{h}_{\bar{x}}-\frac{1}{2} \bar{\tau}_{\bar{x}} \bar{y}^{2} .
\end{aligned}
$$

where the boundary conditions $\bar{u}=\bar{v}=0$ on $\bar{y}=0$ (no-slip and no-penetration) have been imposed, and it has been assumed that the gas core flow exerts a shear stress $\tau=(\mu U / \varepsilon L) \bar{\tau}$ at the free surface $h(x)=\varepsilon L \bar{h}(\bar{x})$ so that $\bar{u}_{\bar{y}}=\bar{\tau}$ on $\bar{y}=\bar{h}(\bar{x})$. The pressure $\bar{p}$ is (as usual in lubrication theory) a function of $\bar{x}$ alone, and is assumed to be continuous across the free surface. If required, the influence of surface tension could be accounted for. However, some simple dimensional analysis shows not only that such effects are small, but also that their inclusion significantly complicates the problem by increasing the number of derivatives by two (though see the discussion at the end of Section 3. below).

Some further discussion of the shear stress term is warranted: we shall assume here for simplicity that $\tau$ is a known constant. Many approximations to this quantity have previously been adopted in the literature. If we set $\tau=c_{f} \rho_{\infty} U_{\infty}^{2} / 2$ then in the current thin layer approximation the constant $c_{f} \geqslant 0$ is the average coefficient of friction (skin friction) of steam on the wet pipe wall. In addition to the wellknown laws $c_{f} \sim 1.328\left(R e_{L}\right)^{-1 / 2}$ and $c_{f} \sim 0.074\left(R e_{L}\right)^{-1 / 5}$ for laminar and turbulent flows respectively (where $R e_{L}=L U_{\infty} \rho_{\infty} / \mu_{g}$ ) many other correlations are available for $c_{f}$ (see also (King \& Tuck, 1993), (Sadatomi et al., 1993) and (Thwaites, 1960)). In the general case a host of other factors such as heat transfer, wall roughness and transition must be taken into account, but in all cases it appears that the shear stress contributes to the leading order equations and must therefore be retained.

We note also that we have ignored the very complicated question of whether the shear stress exerted by gas core flow will cause waves on the surface of the annular film layer. If it does, the the undercutting of these waves will inevitably lead to droplet entrainment. Entrainment of this sort in annular two-phase flows has been both theoretically and experimentally examined by a number of authors (see, for example (Sawant et al., 2009)). Though such a study is outside the scope of this paper, entrainment is clearly an important effect.

\subsection{Mass exchange at the free surface}

At the vapour/liquid interface $y=h(x)$ a transfer of mass takes place as fluid in the liquid layer evaporates to become steam. The details of this evaporative process must be determined as part of the solution to the problem. We assume that the gas/liquid interface is at the saturation temperature $T_{s}$ which is slightly less than the pipe wall temperature. The liquid in the film layer is thus superheated (see, for example, (Higuera, 1987), (Kirillov et al., I), (Kirillov et al., II), (Prosperetti \& Plesset, 1984)) and must cool slightly before it can evaporate. Previous studies have noted that evaporation is not the only way in which mass transfer between the liquid film and the gas can take place. Both droplet deposition from the gas core and the undercutting of any small waves present on the free surface and subsequent entrainment may add to or subtract from evaporative mass exchange. Deposition, as one might expect, seems to be directly proportional to the concentration of liquid droplets in the gas core (see (Fisher \& Pearce, 1993), (Whalley, 1977) and (Whalley, 1987)); mass entrainment is an altogether more complicated phenomenon. At the operating conditions of interest the results of (Collier, 1972) suggest that neither entrainment nor deposition occurs rapidly. Therefore, we assume the dryout phenomenon is dominated by evaporation of the liquid film and ignore all other effects.

Denoting the mass per unit area per unit time transferred from the liquid to the gas by $\dot{M}$ (so that we expect $\dot{M}>0$ ), and assuming that any mass escaping at the free surface does so in the direction of the 


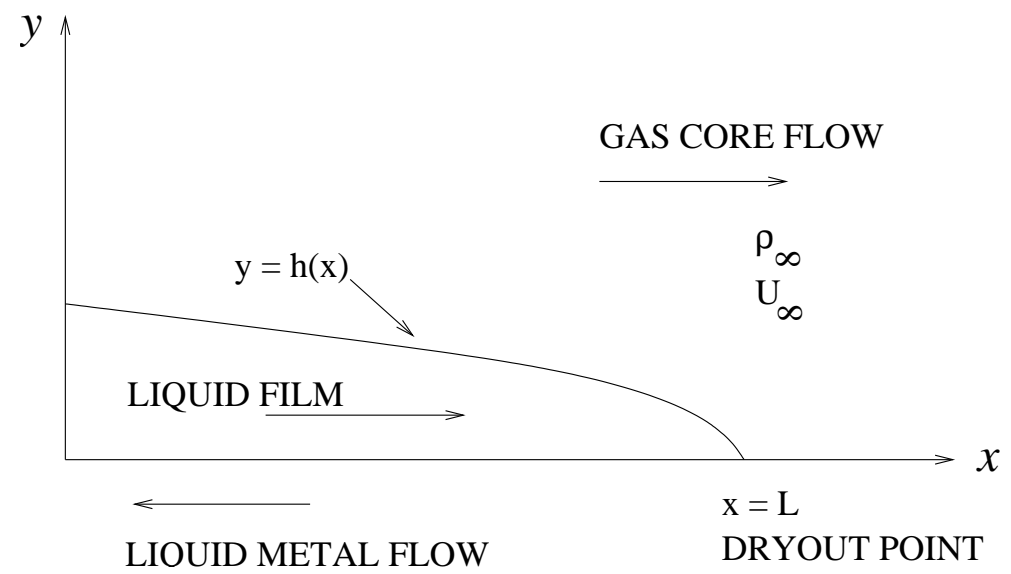

FIG. 2. Schematic diagram of gas/liquid interface and free stream flow.

unit outward-pointing normal $\underline{\hat{n}}$, we have, at the free surface,

$$
\dot{M}=\rho \underline{q} \cdot \underline{\hat{n}}
$$

and hence for steady flow

$$
\dot{M}=\rho\left(-u h_{x}+v\right)\left(1+h_{x}^{2}\right)^{-1 / 2} .
$$

In scaled variables $(2 . .3)$ becomes, to leading order,

$$
\dot{m}=-\bar{u} \bar{h}_{\bar{x}}+\bar{v}
$$

where the nondimensional mass flow rate $\dot{m}$ has been defined by $\dot{M}=\rho U \varepsilon \dot{m}$. This scaling effectively fixes the order of magnitude of the mass transfer required to produce dryout in an $O(L)$ distance. If the mass transfer is an order of magnitude less than this dryout will never occur, and if it is greater then no region of annular flow can exist and the liquid film is instantly vaporised. Use of (2..1) and (2..2) now yields

$$
\dot{m}=\left(\frac{1}{3} \bar{p}_{\bar{x}} \bar{h}^{3}-\frac{1}{2} \bar{\tau} \bar{h}^{2}\right)_{\bar{x}},
$$

or, in dimensional form,

$$
\dot{M}=\frac{\rho}{\mu}\left(\frac{1}{3} p_{x} h^{3}-\frac{1}{2} \tau h^{2}\right)_{x} .
$$

\subsection{Gas core flow}

In the fast-flowing gas core, we assume that the Reynolds number is high, and the flow is incompressible, inviscid and irrotational. We also assume that the gas is a single-phase flow, thereby ignoring any entrained droplets. We denote variables in the gas using a subscript $\mathrm{g}$ and non-dimensionalise according to $x=L \tilde{x}, y=L \tilde{y}, p_{g}=\rho_{\infty} U_{\infty}^{2} \tilde{p}_{g}, h=\varepsilon L \tilde{h}$ and $\phi=L U_{\infty} \tilde{\phi}$ where a tilde denotes a non-dimensional quantity, $\phi$ is the velocity potential of the flow and $\rho_{\infty}$ and $U_{\infty}$ denote respectively a typical speed and 
density of the gas upstream of dryout and therefore far away from the perturbation caused by the liquid film. A schematic diagram of the flow is shown in figure 2.

We now proceed according to standard thin aerofoil theory (for further details see, for example (Van Dyke, 1975)). We assume that the flow is essentially a perturbed plug flow and seek a velocity potential of the form

$$
\tilde{\phi}(\tilde{x}, \tilde{y} ; \varepsilon)=\tilde{x}+\frac{\varepsilon}{2 \pi} \int_{-\infty}^{\infty} f(\xi) \ln \left((\tilde{x}-\xi)^{2}+\tilde{y}^{2}\right) d \xi
$$

where $f(\tilde{x})$ is a distribution of sources along the $\tilde{x}$ axis that has to be determined. We find that, in order to satisfy the linearized thin aerofoil condition $\tilde{\phi}_{\tilde{y}}=\tilde{\phi}_{\tilde{x}} \tilde{h}_{\tilde{x}}$ on $\tilde{y}=0$, we must choose $f(\xi)=\tilde{h}_{\xi}(\xi)$. Proceeding along standard lines and using Bernoulli's equation to relate pressure to velocity in the gas core flow, we find that, in dimensional form, the free stream pressure is given by

$$
p_{g}=p_{\infty}+\frac{\rho_{\infty} U_{\infty}^{2}}{\pi} f_{0}^{L} \frac{h_{\xi}(\xi)}{\xi-x} d \xi
$$

where, as usual, the bar denotes that the integral is of Cauchy principal value type and we have assumed that $h^{\prime}(x)=0$ for $x \leqslant 0$.

\subsection{Heat transfer}

It now only remains to close the model by considering the heat transfer problem in the liquid layer. We non-dimensionalise in the liquid film by setting $x=L \bar{x}, y=\varepsilon L \bar{y}, u=U \bar{u}, v=\varepsilon U \bar{v}$ and $T=T_{s}+$ $\left(T_{w}-T_{s}\right) \bar{T}$ where $T_{s}$ is the saturation temperature of the liquid in the film and $T_{w}$ a typical pipe wall temperature. In the absence of viscous dissipation (which may easily be shown to be negligible) we therefore have, for steady flow,

$$
\bar{u} \bar{T}_{\bar{x}}+\bar{v} \bar{T}_{\bar{y}}=\frac{k}{L \rho U c_{p}}\left(\bar{T}_{\bar{x} \bar{x}}+\frac{1}{\varepsilon^{2}} \bar{T}_{\bar{y} \bar{y}}\right)
$$

where $k$ and $c_{p}$ are respectively the thermal conductivity and specific heat at constant pressure of the liquid (which, for the purposes of this study, we assume are independent of temperature). Using the parameter values in Appendix A (with a liquid layer thickness of $0.5 \mathrm{~mm}$ ), we find that

$$
\frac{k}{L \rho U c_{p} \varepsilon^{2}} \sim 10^{2}, \quad \frac{k}{L \rho U c_{p}} \sim 10^{-6}
$$

and thus to leading order $\bar{T}_{\bar{y} \bar{y}}=0$ and the temperature is simply linear across the liquid layer. There are now many ways to proceed. The wall is heated by a countercurrent flow of liquid metal, and though complicated models may be proposed to couple this flow to the evaporation and the heating of the pipe wall it is then only possible to make progress numerically. Here, we shall assume simply that the wall temperature is maintained at $T_{w}$. Since $T=T_{s}$ at the phase change boundary, this gives

$$
\bar{T}=1-\frac{\bar{y}}{\bar{h}} \text {. }
$$

The change of phase at the liquid/vapour interface must now be accounted for. Assuming that the temperature in the gas core remains constant and neglecting the surface entropy and vapour recoil terms 
(which may easily be shown to be negligible), the standard Stefan condition (see, for example (Rubinstein, 1971)) asserts that

$$
\left[k T_{y}\right]_{\text {liquid }}^{\text {gas }}=\lambda \rho \frac{D}{D t}(y-h(x)),
$$

where the square brackets indicate the jump in the quantity that they enclose, $\lambda$ is the latent heat of vapourisation of the liquid (typically $\lambda \sim 600 \mathrm{~kJ} / \mathrm{kg}$ ) and $D / D t=\partial / \partial t+q \underline{q} . \nabla$ is the usual material derivative. Using the nondimensional scalings introduced earlier, we find that, for steady flow,

$$
\left.\bar{T}_{\bar{y}}\right|_{\text {liquid }}=-\frac{1}{\bar{\eta}}\left(\bar{v}-\bar{u} \bar{h}_{\bar{x}}\right)
$$

where $\bar{\eta}=k L\left(T_{w}-T_{s}\right) /\left(U \rho \lambda h_{0}^{2}\right)$ is a nondimensional parameter that characterises the transfer of mass from the liquid film into the gas core. Note that since by assumption only evaporation (and not condensation) is taking place and the liquid is superheated, necessarily $\bar{\eta}>0$. Also, the definition of $\bar{\eta}$ predictably implies that the mass transfer increases with increasing thermal conductivity and amounts of superheat, but decreases with increasing liquid flow speed, liquid density and liquid latent heat.

From (2..4) we have $\dot{m}=-\bar{u} \bar{h}_{\bar{x}}+\bar{v}$, and thus

$$
-\dot{m}=\left.\bar{\eta} \bar{T}_{\bar{y}}\right|_{\text {liquid }}
$$

so that

$$
\dot{m}=\frac{\bar{\eta}}{\bar{h}}
$$

In dimensional form, (2..7) becomes

$$
\dot{M}=\frac{k\left(T_{w}-T_{s}\right)}{\lambda h} .
$$

This equation may be thought of as a mass transfer constitutive law that relates the interfacial mass flux $\dot{M}$ to the temperature of the pipe wall and the other physical variables in the problem. For the operating conditions of interest, the typical parameter values given in Appendix A imply that, for a fluid layer of thickness $1 \mathrm{~mm}$,

$$
\bar{\eta} \sim 0.7\left(T_{w}-T_{s}\right) / K
$$

indicating that, with a few degrees of superheat in the liquid, dryout will, as assumed, occur at an $O(1)$ distance from the onset of the annular flow.

A similar simple heat transfer analysis may also be carried out when the wall heat flux (rather than the wall temperature) is known. This gives $\dot{m}=\hat{\eta}$ where $\hat{\eta}=L q /\left(\lambda \rho U h_{0}\right)$ and $q$ denotes the wall heat transfer so that $\left.k T_{y}\right|_{y=0}=-q$. Many other boundary conditions at the liquid metal/pipe wall interface may also be modelled. For simplicity, however, we shall henceforth consider only the mass transfer equation (2..7).

\subsection{Integral equation}

It is now possible to close the model. Using (2..5), (2..6), (2..8), and the fact that $p=p_{g}$ at the liquid/vapour interface, we conclude that, in dimensional variables,

$$
\left[\frac{h^{3}}{3}\left(\frac{\rho_{\infty} U_{\infty}^{2}}{\pi} f_{0}^{L} \frac{h_{\xi}(\xi)}{\xi-x} d \xi\right)_{x}-\frac{h^{2} \tau}{2}\right]_{x}=\frac{\mu k\left(T_{w}-T_{s}\right)}{\lambda \rho h} .
$$


This equation is more conveniently posed in terms of the nondimensional variables defined earlier. We find that

$$
\left[\bar{\theta} \frac{\bar{h}^{3}}{3}\left(\frac{1}{\pi} f_{0}^{1} \frac{\bar{h}_{\xi}(\xi)}{\xi-\bar{x}} d \xi\right)_{\bar{x}}-\frac{\bar{h}^{2}}{2} \bar{\tau}\right]_{\bar{x}}=\frac{\bar{\eta}}{\bar{h}} .
$$

where $\bar{\theta}=\varepsilon^{3} L \rho_{\infty} U_{\infty}^{2} /(\mu U)$ characterises the relative importance of the pressure variations in the gas core to those in the liquid layer. Using the values in Appendix A with a liquid layer thickness of $1 \mathrm{~mm}$, we find that $\bar{\theta} \sim 1.5$, indicating that the Cauchy integral term is a leading-order effect that must be retained.

Some thought must be given both to the specification of boundary conditions for (2..9), and to how the problem may be set up to determine $L$. We note that (2..9) is a third order nonlinear singular integrodifferential equation (NSIDE). There appears to be virtually no rigorous existence and uniqueness theory in the literature for such equations, and it is not immediately clear how many boundary conditions will be required to solve the equation and determine $L$, the length to dryout. Proceeding on the basis however that an $n$th order singular integro-differential equation normally requires $n+1$ boundary conditions (one for each order and an "inversion" condition - see, for example (Cuminato et al., 2007)) it seems sensible to impose the obvious boundary conditions on (2..9). These are

$$
\bar{h}(0)=1, \quad \bar{h}(1)=0, \quad \bar{h}^{\prime}(0)=0
$$

and

$$
\left[\bar{\theta} \frac{\bar{h}^{3}}{3}\left(\frac{1}{\pi} f_{0}^{1} \frac{\bar{h}_{\xi}(\xi)}{\xi-\bar{x}} d \xi\right)_{\bar{x}}-\frac{\bar{h}^{2}}{2} \bar{\tau}\right]_{\bar{x}=1}=0 .
$$

The first two of these conditions reflect the geometry of the problem, and the third insists that the pressure is finite at the onset of annular flow. The final condition (2..11) expresses the fact that the mass flux from the liquid film must be zero at the dryout point.

We shall see below that the boundary conditions (2..10) and (2..11) appear to be enough to specify a unique solution to (2..9). We shall also see in the next Section that, as might be expected, an additional boundary condition is required to determine the length $L$ to dryout. It is also worth noting that (2..9) depends only upon the two non-dimensional parameters $\bar{\tau} / \bar{\theta}$ and $\bar{\eta} / \bar{\theta}$.

\subsection{Paradigm problem}

Although the asymptotic and numerical solution of (2..9) with the boundary conditions (2..10) and (2..11) will be the subject of most of the remainder of this study, we pause at this stage to consider whether the boundary conditions (2..10) and (2..11) are appropriate for the problem and how $L$ may be determined. Since (2..9) is awkward to work with, we shall make, for illustrative purposes, the (physically untenable) assumptions that $\bar{\tau} \sim 2 \bar{\theta} \tau_{0}^{*} \bar{x} \bar{h}^{-2}$ and $\bar{\eta} \sim \eta_{0}^{*} \bar{h} \bar{\theta}$ and ignore the $\bar{h}^{3} / 3$ term multiplying the singular integral term in (2..9). The problem then becomes

$$
\left[\left(\frac{1}{\pi} f_{0}^{1} \frac{\bar{h}_{\xi}(\xi)}{\xi-\bar{x}} d \xi\right)_{\bar{x}}-\tau_{0}^{*} \bar{x}\right]_{\bar{x}}=\eta_{0}^{*}
$$

where $\eta_{0}^{*}$ and $\tau_{0}^{*}$ are constants. This paradigm problem, which will prove to be useful from a theoretical, asymptotic and numerical point of view, may be solved in closed form. By integrating and using the 
obvious analogous "mass flow" condition to (2..11), we find that

$$
\left(\frac{1}{\pi} f_{0}^{1} \frac{\bar{h}_{\xi}(\xi)}{\xi-\bar{x}} d \xi\right)_{\bar{x}}-\tau_{0}^{*} \bar{x}=\eta_{0}^{*}(\bar{x}-1)
$$

Further rearranging, integrating, inverting using standard methods (see, for example (Muskhelishvili, 1953)), applying the boundary condition $\bar{h}^{\prime}(0)=0$ and finally integrating again and applying $\bar{h}(0)=1$ and $\bar{h}(1)=0$, we thus have

$$
\bar{h}(\bar{x})=\frac{1}{48} \sqrt{\bar{x}(1-\bar{x})}\left[-16 K \bar{x}^{2}+\bar{x}\left(24 \eta_{0}^{*}-8 K\right)+\frac{96}{\pi}\right]-\frac{1}{\pi} \sin ^{-1}(2 \bar{x}-1)+\frac{1}{2} \quad(0 \leqslant x \leqslant 1) .
$$

where $K=\left(\tau_{0}^{*}+\eta_{0}^{*}\right) / 2$. It may easily be shown that (2..14) is the unique solution to (2..12) that satisfies the conditions (2..10) and the analogous condition to (2..11); the nonlinear nature of (2..9) makes it unlikely, however, that it will ever be possible to establish uniqueness of the solution to the full problem.

We must now consider how to accomplish the major objective of this study and determine the length $L$ of the dryout region. Clearly another boundary condition is required. Many specifications of the problem are possible, but we shall assume here that the pressure $p_{g 0}$ is known at the onset of annular flow $\bar{x}=0$. This condition is equivalent to prescribing the total mass flux in the pipe: for a positive mass flux we require $p_{g 0}<p_{\infty}$. Thus

$$
p_{g 0}=p_{\infty}+\frac{\varepsilon \rho_{\infty} U_{\infty}^{2}}{\pi} f_{0}^{1} \frac{\bar{h}_{\xi}(\xi)}{\xi} d \xi
$$

using the solution (2..14), we find that

$$
\frac{p_{g 0}-p_{\infty}}{\varepsilon \rho_{\infty} U_{\infty}^{2}}=\frac{1}{\pi}\left[-2+\frac{3 \eta_{0}^{*} \pi}{16}-\frac{\tau_{0}^{*} \pi}{16}\right]
$$

and thus, for the paradigm problem,

$$
L=\frac{h_{0} \rho_{\infty} U_{\infty}^{2}}{16\left(p_{\infty}-p_{g 0}\right)}\left[\frac{32}{\pi}-3 \eta_{0}^{*}+\tau_{0}^{*}\right]
$$

Since the quantity $p_{\infty}-p_{g 0}$ is positive, the dryout length predicted by $(2 . .15)$ is positive so long as $\eta_{0}^{*}$ is not too large. For later reference, we note that, as far as the behaviour of (2..14) is concerned,

1. Near $x=0$, though $h(0)=1$ and $h^{\prime}(0)=0$, we have $h^{\prime \prime}(0) \sim\left(5 \pi \eta_{0}^{*}-\pi \tau_{0}^{*}-16\right) /(16 \pi \sqrt{x})$. The solution is therefore non-monotone with $h(0+)>1$ if $\eta_{0}^{*}>\tau_{0}^{*} / 5+16 /(5 \pi)$.

2. Near $x=1$, we have $h^{\prime}(x) \sim\left(\pi\left(\tau_{0}^{*}-\eta_{0}^{*}\right)-16\right) /(8 \pi \sqrt{1-x})$, so that $h(1-)$ will be negative (and the solution will therefore be unphysical) if $\tau_{0}^{*}>\eta_{0}^{*}+16 / \pi$.

3. We note that $L$ increases as $\tau_{0}^{*}$ increases and decreases as $\eta_{0}^{*}$ increases, both these results being physically reasonable. When the heat transfer is so large that $3 \eta_{0}^{*}>\tau_{0}^{*}+32 / \pi$, the quantity $L$ becomes negative and the solution again breaks down. 
Though an exact solution was available for the paradigm problem, it may also be solved numerically. For brevity, the details are not given here. Although there are many accurate and efficient methods for solving linear singular integro-differential equations (see, for example (Anderssen et al., 1980), (Golberg, 1979) and (Krenk, 1975)), it is better to eschew such methods as they rely specifically on the linearity of the equation - and (2..9) is nonlinear. In solving (2..12), two matters immediately become clear: first, regularization is crucial to deal with the conditions at $x=0$ and $x=1$ and second, knowledge of the asymptotic behaviour of the solution is extremely helpful for the development of good numerical schemes. We now deal with these matters for the full equation.

\section{Asymptotic analysis of the governing equation}

Following the discussion of the previous Section, our first task is to determine the asymptotic behaviour of solutions to the full problem

$$
\left[\bar{\theta} \frac{\bar{h}^{3}}{3}\left(\frac{1}{\pi} f_{0}^{1} \frac{\bar{h}_{\xi}(\xi)}{\xi-\bar{x}} d \xi\right)_{\bar{x}}-\frac{\bar{h}^{2}}{2} \bar{\tau}\right]_{\bar{x}}=\frac{\bar{\eta}}{\bar{h}}
$$

with boundary conditions

$$
\bar{h}(0)=1, \quad \bar{h}(1)=0, \quad \bar{h}^{\prime}(0)=0, \quad\left[\bar{\theta} \frac{\bar{h}^{3}}{3}\left(\frac{1}{\pi} f_{0}^{1} \frac{\bar{h}_{\xi}(\xi)}{\xi-\bar{x}} d \xi\right)_{\bar{x}}-\frac{\bar{h}^{2}}{2} \bar{\tau}\right]_{\bar{x}=1}=0 .
$$

There is little interest in the asymptotic behaviour of solutions near to the point $\bar{x}=0$, for here $\bar{h}_{\bar{x}}$ is zero and there are no numerical difficulties. (It is relatively easy to show, however, that for $\bar{x} \ll 1$, $\bar{h}(\bar{x}) \sim 1-O\left(\bar{x}^{3 / 2}\right)$.) At the dryout point, however, $\bar{h}$ is zero and, as suggested by the paradigm problem, much more care is required. Here is evident that the term $\bar{\eta} / \bar{h}$ on the right hand side of (3..1) must balance with the first term on the left hand side of the equation. Assuming that $\bar{h}(\bar{x}) \sim \bar{A}(1-\bar{x})^{p}$ as $\bar{x} \rightarrow 1$ (where $\bar{A}$ is a positive constant and $0<p<1$ ) we have

$$
\frac{\eta}{\bar{A}(1-\bar{x})^{p}} \sim\left[\frac{\bar{\theta} \bar{A}^{3}(1-\bar{x})^{3 p}}{3 \pi}\left(f_{0}^{1} \frac{\bar{h}_{\xi}(\xi)}{\xi-\bar{x}} d \xi\right)_{\bar{x}}\right]_{\bar{x}} \quad(\bar{x} \sim 1)
$$

Now consider

$$
I=f_{0}^{1} \frac{\bar{h}_{\xi}(\xi)}{\xi-\bar{x}} d \xi \sim \int_{0}^{R} \frac{\bar{h}_{\xi}(\xi)}{\xi-\bar{x}} d \xi+f_{R}^{1} \frac{-p \bar{A}(1-\xi)^{p-1}}{\xi-\bar{x}} d \xi \quad(\bar{x} \sim 1)
$$

where the constant $R<1$ is chosen so that $1-R \ll 1$ but $1-x \ll 1-R$. Rearranging, we find that

$$
I \sim \int_{0}^{R} \frac{\bar{h}_{\xi}+\bar{A} p(1-\xi)^{p-1}}{\xi-\bar{x}} d \xi-f_{0}^{1} \frac{\bar{A} p(1-\xi)^{p-1}}{\xi-\bar{x}} d \xi
$$

and, on setting $1-\xi=(1-\bar{x}) u$ in the principal value integral, we obtain

$$
I \sim \int_{0}^{R} \frac{\bar{h}_{\xi}+\bar{A} p(1-\xi)^{p-1}}{\xi-\bar{x}} d \xi-\bar{A} p(1-\bar{x})^{p-1}\left[f_{0}^{\infty} \frac{u^{p-1}}{1-u} d u-\int_{\frac{1}{1-\bar{x}}}^{\infty} \frac{u^{p-1}}{1-u} d u\right] .
$$


Now using the standard result

$$
f_{0}^{\infty} \frac{u^{p-1}}{1-u} d u=\pi \cot p \pi \quad(0<p<1)
$$

we conclude that, provided $\bar{h}_{\xi}$ is well-behaved away from $\bar{x}=1$ (which is, in any case, a physically sensible requirement), then, to leading order,

$$
I \sim-\bar{A} p \pi \cot p \pi(1-\bar{x})^{p-1} \quad(\bar{x} \sim 1) .
$$

The equation (3..3) now yields $p=3 / 5$ and thus, near the dryout point $\bar{x}=1$, we have

$$
\bar{h} \sim \bar{A}(1-\bar{x})^{3 / 5}
$$

where

$$
\bar{A}=\left(\frac{-125 \tan (3 \pi / 5) \bar{\eta}}{4 \bar{\theta}}\right)^{\frac{1}{5}} \sim 2.49\left(\frac{\bar{\eta}}{\bar{\theta}}\right)^{1 / 5} .
$$

Returning for a moment to dimensional variables, we find that, for $x \sim L$,

$$
h^{\prime}(L) \sim-\Gamma\left(\frac{k\left(T_{w}-T_{S}\right) v}{\lambda \rho_{\infty} U_{\infty}^{2}}\right)^{1 / 5}(L-x)^{-2 / 5}
$$

where $\Gamma \simeq 3 / 2$. The dependence of the gradient on the problem parameters is therefore as one might expect, for the length of the fluid region presumably increases with increasing $\lambda$ (the greater the latent heat the harder it is to evaporate the fluid) and $\rho_{\infty} U_{\infty}^{2}$ (the free stream has more power), but decreases with increasing $k\left(T_{w}-T_{S}\right)$ (more wall heating) and $v$ (the fluid is more viscous and is therefore less inclined to flow). All of these observations are correctly predicted by (3..5), since as $L$ increases we expect the gradient near to $x=L$ to decrease.

It is worth pointing out that, unlike in some previous studies (see, for example (King \& Tuck, 1993)), the infinite gradient of the solution at $\bar{x}=1$ is essentially a consequence of the evaporation model and cannot be removed simply by including the effects of surface tension. Specifically, inclusion of this term would lead to an asymptotic balance between $\bar{S}\left(\bar{h}^{3} \bar{h}_{\bar{x} \bar{x} \bar{x}}\right)_{\bar{x}} / 3$ (where $\bar{S}=\varepsilon^{3} \sigma /(\mu U)$ and $\sigma(\mathrm{N} / \mathrm{m})$ is the fluid surface tension) and $\bar{\eta} / \bar{h}$ near $\bar{x}=1$. This gives $\bar{h} \sim(1-\bar{x})^{4 / 5}$ and thus slightly reduces the strength of (but does not eliminate) the slope singularity. Note also that for the case of constant wall heat flux it is easy to show that $\bar{h}_{\bar{x}}$ is still infinite at the dryout point. We therefore continue to ignore the effects of surface tension since, in any case, using the values in Appendix A, we have $\bar{S} \ll 1$.

\section{Problem reformulation and regularization}

To allow a numerical scheme to be implemented, the equation (3..1) and its associated boundary conditions (3..2) must be both reformulated and regularised. Integrating and using the mass flux condition at $\bar{x}=1$, before rearranging and integrating once again, we have

$$
\frac{1}{\pi} \int_{0}^{1} \frac{\bar{h}_{\xi}(\xi)}{\xi-\bar{x}} d \xi=\frac{3 \bar{\tau}}{2 \bar{\theta}} \int_{0}^{\bar{x}} \frac{1}{\bar{h}(\xi)} d \xi-\frac{3 \bar{\eta}}{\bar{\theta}} \int_{0}^{\bar{x}}\left(\frac{1}{\bar{h}^{3}(\xi)} \int_{\xi}^{1} \frac{1}{\bar{h}(s)} d s\right) d \xi+C_{2}
$$

We now define

$$
F(\bar{x}, \bar{h}(\bar{x}))=\frac{3 \bar{\tau}}{2 \bar{\theta}} \int_{0}^{\bar{x}} \frac{1}{\bar{h}(\xi)} d \xi-\frac{3 \bar{\eta}}{\bar{\theta}} \int_{0}^{\bar{x}}\left(\frac{1}{\bar{h}^{3}(\xi)} \int_{\xi}^{1} \frac{1}{\bar{h}(s)} d s\right) d \xi
$$


so that (4..1) may be rewritten as

$$
\frac{1}{\pi} f_{0}^{1} \frac{\bar{h}_{\xi}(\xi)}{\xi-\bar{x}} d \xi=F(\bar{x}, \bar{h}(\bar{x}))+C_{2} .
$$

By using standard inversion formulae for Cauchy integral equations (see, for example (Muskhelishvili, 1953)), (4..2) may be inverted to give

$$
\begin{gathered}
\bar{h}_{\bar{x}}(\bar{x})=-\frac{1}{\pi \sqrt{\bar{x}(1-\bar{x})}} \int_{0}^{1} \frac{\sqrt{\xi(1-\xi)} F(\xi, \bar{h}(\xi))}{\xi-\bar{x}} d \xi- \\
\frac{C_{2}}{\pi \sqrt{\bar{x}(1-\bar{x})}} \int_{0}^{1} \frac{\sqrt{\xi(1-\xi)}}{\xi-\bar{x}} d \xi+\frac{C_{3}}{\sqrt{\bar{x}(1-\bar{x})}} .
\end{gathered}
$$

Now using the fact that

$$
f_{0}^{1} \frac{\sqrt{\xi(1-\xi)}}{\xi-\bar{x}} d \xi=-\pi \bar{x}+\frac{\pi}{2} \quad(0<\bar{x}<1)
$$

and imposing the boundary condition $\bar{h}_{\bar{x}}(0)=0$, we find that

$$
\begin{aligned}
& \bar{h}_{\bar{x}}(\bar{x})=-\frac{1}{\pi \sqrt{\bar{x}(1-\bar{x})}} f_{0}^{1} \frac{\sqrt{\xi(1-\xi)} F(\xi, \bar{h}(\xi))}{\xi-\bar{x}} d \xi+ \\
& \frac{(1-2 \bar{x})}{\pi \sqrt{\bar{x}(1-\bar{x})}} f_{0}^{1} \frac{\sqrt{\xi(1-\xi)} F(\xi, \bar{h}(\xi))}{\xi} d \xi+\frac{2 \bar{x} C_{3}}{\sqrt{\bar{x}(1-\bar{x})}} .
\end{aligned}
$$

Integrating once again, using the boundary condition $\bar{h}(0)=1$ and changing the order of integration, we find that

$$
\begin{aligned}
\bar{h}(\bar{x})=-\frac{1}{\pi} & \int_{0}^{1}\left(F(\xi, \bar{h}(\xi)) \sqrt{\xi(1-\xi)} \int_{0}^{\bar{x}} \frac{d s}{(\xi-s) \sqrt{s(1-s)}}\right) d \xi+ \\
& \frac{2}{\pi} \sqrt{\bar{x}(1-\bar{x})} \int_{0}^{1} \frac{\sqrt{\xi(1-\xi)} F(\xi, \bar{h}(\xi))}{\xi} d \xi+ \\
& 2 C_{3}\left(-\sqrt{\bar{x}(1-\bar{x})}+\frac{1}{2} \sin ^{-1}(2 \bar{x}-1)+\frac{\pi}{4}\right)+1 .
\end{aligned}
$$

Using the standard integral

$$
\sqrt{\xi(1-\xi)} \int_{0}^{\bar{x}} \frac{d s}{(\xi-s) \sqrt{s(1-s)}}=\ln \left|\frac{-\xi-\bar{x}+2 \xi \bar{x}-2 \sqrt{\xi(1-\xi)} \sqrt{\bar{x}(1-\bar{x})}}{\xi-\bar{x}}\right|
$$

and imposing the remaining boundary condition $\bar{h}(1)=0$, we obtain $C_{3}=-1 / \pi$ so that

$$
\begin{gathered}
\bar{h}(\bar{x})=-\frac{1}{\pi} \int_{0}^{1} F(\xi, \bar{h}(\xi)) \ln \left|\frac{-\xi-\bar{x}+2 \xi \bar{x}-2 \sqrt{\xi(1-\xi)} \sqrt{\bar{x}(1-\bar{x})}}{\xi-\bar{x}}\right| d \xi+ \\
\frac{2}{\pi} \sqrt{\bar{x}(1-\bar{x})} \int_{0}^{1} \frac{\sqrt{\xi(1-\xi)} F(\xi, \bar{h}(\xi))}{\xi} d \xi+\frac{2}{\pi} \sqrt{\bar{x}(1-\bar{x})}-\frac{1}{\pi} \sin ^{-1}(2 \bar{x}-1)+\frac{1}{2} .
\end{gathered}
$$


From a numerical point of view, recasting the problem in this way is advantageous as there are now no singular integrals or derivatives in the equation. The problem may now be regularised by using the asymptotic information that was gathered in Section 3 . by setting $\bar{h}(\bar{x})=\bar{H}(\bar{y})$, where $\bar{y}^{5 / 3}=1-\bar{x}$. We obtain the equation

$$
\begin{gathered}
\bar{H}(\bar{y})=-\frac{5}{3 \pi} \int_{0}^{1} \bar{\xi}^{2 / 3} \bar{F}\left(\left(1-\bar{\xi}^{5 / 3}\right), \bar{H}(\bar{\xi})\right) \times \\
\ln \left|\frac{\bar{\xi}^{5 / 3}+\bar{y}^{5 / 3}-2 \bar{\xi}^{5 / 3} \bar{y}^{5 / 3}+2 \sqrt{\bar{\xi}^{5 / 3}\left(1-\bar{\xi}^{5 / 3}\right)} \sqrt{\bar{y}^{5 / 3}\left(1-\bar{y}^{5 / 3}\right)}}{\bar{\xi}^{5 / 3}-\bar{y}^{5 / 3}}\right| d \bar{\xi}+ \\
\frac{10}{3 \pi} \sqrt{\bar{y}^{5 / 3}\left(1-\bar{y}^{5 / 3}\right)} \int_{0}^{1} \frac{\bar{\xi}^{2 / 3} \bar{F}\left(\left(1-\bar{\xi}^{5 / 3}\right), \bar{H}(\bar{\xi})\right) \sqrt{\bar{\xi}^{5 / 3}\left(1-\bar{\xi}^{5 / 3}\right)}}{1-\bar{\xi}^{5 / 3}} d \bar{\xi}+ \\
\frac{2}{\pi} \sqrt{\bar{y}^{5 / 3}\left(1-\bar{y}^{5 / 3}\right)}-\frac{1}{\pi} \sin ^{-1}\left(1-2 \bar{y}^{5 / 3}\right)+1 / 2 \quad(0 \leqslant \bar{y} \leqslant 1)
\end{gathered}
$$

where

$$
\bar{F}(\bar{y}, \bar{H}(\bar{y}))=\frac{5 \bar{\tau}}{2 \bar{\theta}} \int_{\bar{y}}^{1} \frac{\bar{\xi}^{2 / 3}}{\bar{H}(\bar{\xi})} d \bar{\xi}-\frac{25 \bar{\eta}}{3 \bar{\theta}} \int_{\bar{y}}^{1} \frac{\bar{\xi}^{2 / 3}}{\bar{H}^{3}(\bar{\xi})}\left(\int_{0}^{\bar{\xi}} \frac{\bar{s}^{2 / 3}}{\bar{H}(\bar{s})} d \bar{s}\right) d \bar{\xi}
$$

and, of course, automatically $H(0)=0$ and $H(1)=1$.

\section{Numerical Method and Results}

We now present a numerical procedure for the solution of the final regularized problem (4..3). First, we divide the interval $[0,1]$ into $n$ equally spaced subintervals $\left[\bar{y}_{i}, \bar{y}_{i+1}\right]$, where $1 \leqslant i \leqslant n$ and $\bar{y}_{1}=0$, $\bar{y}_{n+1}=1$. Proceeding by collocation, we discretize (4...3) to obtain

$$
\begin{gathered}
\bar{H}\left(\bar{y}_{i}\right)=-\frac{5}{3 \pi} \sum_{j=1}^{n} \int_{\bar{y}_{j}}^{\bar{y}_{j+1}} \bar{\xi}^{2 / 3} \bar{F}\left(\left(1-\bar{\xi}^{5 / 3}\right), \bar{H}(\bar{\xi})\right) \times \\
\ln \left|\frac{\bar{\xi}^{5 / 3}+\bar{y}_{i}^{5 / 3}-2 \bar{\xi}^{5 / 3} \bar{y}_{i}^{5 / 3}+2 \sqrt{\bar{\xi}^{5 / 3}\left(1-\bar{\xi}^{5 / 3}\right)} \sqrt{\bar{y}_{i}^{5 / 3}\left(1-\bar{y}_{i}^{5 / 3}\right)} \mid d \bar{\xi}^{5 / 3}-\bar{y}_{i}^{5 / 3}}{\bar{\xi}^{5 / 3}}\right|_{j=1}^{n} \int_{\bar{y}_{j}}^{\bar{y}_{j+1}} \frac{\bar{\xi}^{2 / 3} \bar{F}\left(\left(1-\bar{\xi}^{5 / 3}\right), \bar{H}(\bar{\xi})\right) \sqrt{\bar{\xi}^{5 / 3}\left(1-\bar{\xi}^{5 / 3}\right)}}{10} d \bar{\xi}_{+}+ \\
\frac{2}{\pi} \sqrt{\bar{y}_{i}^{5 / 3}\left(1-\bar{y}_{i}^{5 / 3}\right)} \\
\bar{y}_{i}^{5 / 3}\left(1-\bar{y}_{i}^{5 / 3}\right)-\frac{1}{\pi} \sin ^{-1}\left(1-2 \bar{y}_{i}^{5 / 3}\right)+\frac{1}{2} \quad(i=2,3 \ldots n) .
\end{gathered}
$$

To approximate the function $F$, we write

$$
\bar{F}(\bar{\xi}, \bar{H}(\bar{\xi}))=C_{\tau} \int_{\bar{\xi}}^{1} \frac{s^{2 / 3}}{\bar{H}(s)} d s-C_{\eta} \int_{\bar{\xi}}^{1} \frac{s^{2 / 3}}{\bar{H}^{3}(s)}\left(\int_{0}^{s} \frac{t^{2 / 3}}{\bar{H}(t)} d t\right) d s
$$

where $C_{\tau}=\frac{5 \bar{\tau}}{2 \bar{\theta}}$ and $C_{\eta}=\frac{25 \bar{\eta}}{3 \bar{\theta}}$. 
For convenience we henceforth omit the overbars and use a linear approximation for $H$ over each sub-interval $\left[\xi_{i}, \xi_{i+1}\right]$ using $H(\xi) \simeq a_{i} \xi+b_{i}$, where $a_{i}=\frac{H_{i+1}-H_{i}}{d \xi}, b_{i}=H_{i}-\xi_{i} a_{i}, H_{i} \simeq H\left(\xi_{i}\right)$ and $d \xi=$ $\xi_{i+1}-\xi_{i}$. In (5..2), we denote the first integral by $M(\xi)$ and the second (double integral) by $N(\xi)$. For $\xi \in\left[\xi_{j}, \xi_{j+1}\right]$, with $1 \leqslant j<n$, the integral $M$ is approximated by

$$
\begin{gathered}
M(\xi) \simeq \int_{\xi}^{\xi_{j+1}} \frac{s^{2 / 3}}{a_{j} s+b_{j}} d s+\sum_{k=j+1}^{n} \int_{\xi_{k}}^{\xi_{k+1}} \frac{s^{2 / 3}}{a_{k} s+b_{k}} d s \\
=\left[\Psi\left(\xi_{j+1}, a_{j}, b_{j}\right)-\Psi\left(\xi, a_{j}, b_{j}\right)\right]+\sum_{k=j+1}^{n}\left(\Psi\left(\xi_{k+1}, a_{k}, b_{k}\right)-\Psi\left(\xi_{k}, a_{k}, b_{k}\right)\right) .
\end{gathered}
$$

If $j=n$ then (5..3) still holds, but the summation term is ignored. Here and henceforth the functions $\Psi$ and $\Phi$ (which are integrals that can be calculated in closed form) are given by

$$
\Psi(y, a, b)=\int_{0}^{y} \frac{x^{2 / 3}}{a x+b} d x \quad \Phi(y, a, b)=\int_{0}^{y} \frac{x^{2 / 3}}{(a x+b)^{3}} d x .
$$

For $\xi \in\left[\xi_{j}, \xi_{j+1}\right]$, the function $N$ is approximated for $1 \leqslant j<n$ by

$$
\begin{gathered}
N(\xi) \simeq \int_{\xi}^{\xi_{j+1}} \frac{s^{2 / 3}}{\left(a_{j} s+b_{j}\right)^{3}}\left(\int_{0}^{s} \frac{t^{2 / 3}}{H(t)} d t\right) d s+\sum_{k=j+1}^{n} \int_{\xi_{k}}^{\xi_{k+1}} \frac{s^{2 / 3}}{\left(a_{k} s+b_{k}\right)^{3}}\left(\int_{0}^{s} \frac{t^{2 / 3}}{H(t)} d t\right) \\
=\int_{\xi}^{\xi_{j+1}} \frac{s^{2 / 3}}{\left(a_{j} s+b_{j}\right)^{3}}\left[\sum_{k=1}^{j-1}\left(\Psi\left(x_{k+1}, a_{k}, b_{k}\right)-\Psi\left(x_{k}, a_{k}, b_{k}\right)\right)+\Psi\left(s, a_{j}, b_{j}\right)-\Psi\left(x_{j}, a_{j}, b_{j}\right)\right] d s+ \\
\sum_{k=j+1}^{n} \int_{\xi_{k}}^{\xi_{k+1}} \frac{s^{2 / 3}}{\left(a_{k} s+b_{k}\right)^{3}}\left[\sum_{l=1}^{k-1}\left(\Psi\left(x_{l+1}, a_{l}, b_{l}\right)-\Psi\left(x_{l}, a_{l}, b_{l}\right)\right)+\Psi\left(s, a_{k}, b_{k}\right)-\Psi\left(x_{k}, a_{k}, b_{k}\right)\right] d s \\
=\left(\sum_{k=1}^{j-1} \Psi\left(x_{k+1}, a_{k}, b_{k}\right)-\Psi\left(x_{k}, a_{k}, b_{k}\right)\right)\left(\Phi\left(\xi_{j+1}, a_{j}, b_{j}\right)-\Phi\left(\xi, a_{j}, b_{j}\right)\right)+ \\
\sum_{\xi}^{\xi_{j+1}} \frac{s^{2 / 3}}{\left(a_{j} s+b_{j}\right)^{3}}\left(\Psi\left(s, a_{j}, b_{j}\right)-\Psi\left(x_{j}, a_{j}, b_{j}\right)\right) d s+ \\
\sum_{k=j+1}^{n}\left(\sum_{l=1}^{k-1} \Psi\left(x_{l+1}, a_{l}, b_{l}\right)-\Psi\left(x_{l}, a_{l}, b_{l}\right)\right)\left(\Phi\left(x_{k+1}, a_{k}, b_{k}\right)-\Phi\left(x_{k}, a_{k}, b_{k}\right)\right)+ \\
\sum_{k=j+1}^{n} \int_{\xi_{k}}^{\xi_{k+1}} \frac{s^{2 / 3}}{\left(a_{k} s+b_{k}\right)^{3}}\left(\Psi\left(s, a_{k}, b_{k}\right)-\Psi\left(x_{k}, a_{k}, b_{k}\right)\right) d s
\end{gathered}
$$

where once again the relevant summation terms should be ignored if $j=n$.

\subsection{Numerical Results}

The expression (5..1) may now be used to define a direct iteration method to solve (4..3). If we start the iterative process by assuming that $H$ is linear, then numerical experiments quickly suggest that convergence is easily obtained, though because of the nonlinear nature of the problem it appears unlikely that rigorous convergence results are likely to be available. Notwithstanding this, for each of the illustrative 
numerical results that are discussed below, all the usual tests were performed to ensure that the final solution was insensitive to the choice of initial approximation, the solution converged in a satisfactory manner as the number of grid points was increased, and the known asymptotic properties of the solution were faithfully reproduced (the details are omitted for brevity).

We note that the numerical solution depends only upon the parameters $C_{\tau}$ and $C_{\eta}$. Numerical experiments show that it is not possible to compute a numerical solution to the problem for all parameter pairs $\left(C_{\tau}, C_{\eta}\right)$. The figures below show some representative results and how the numerical solution varies for various values of $C_{\tau}$ and $C_{\eta}$.

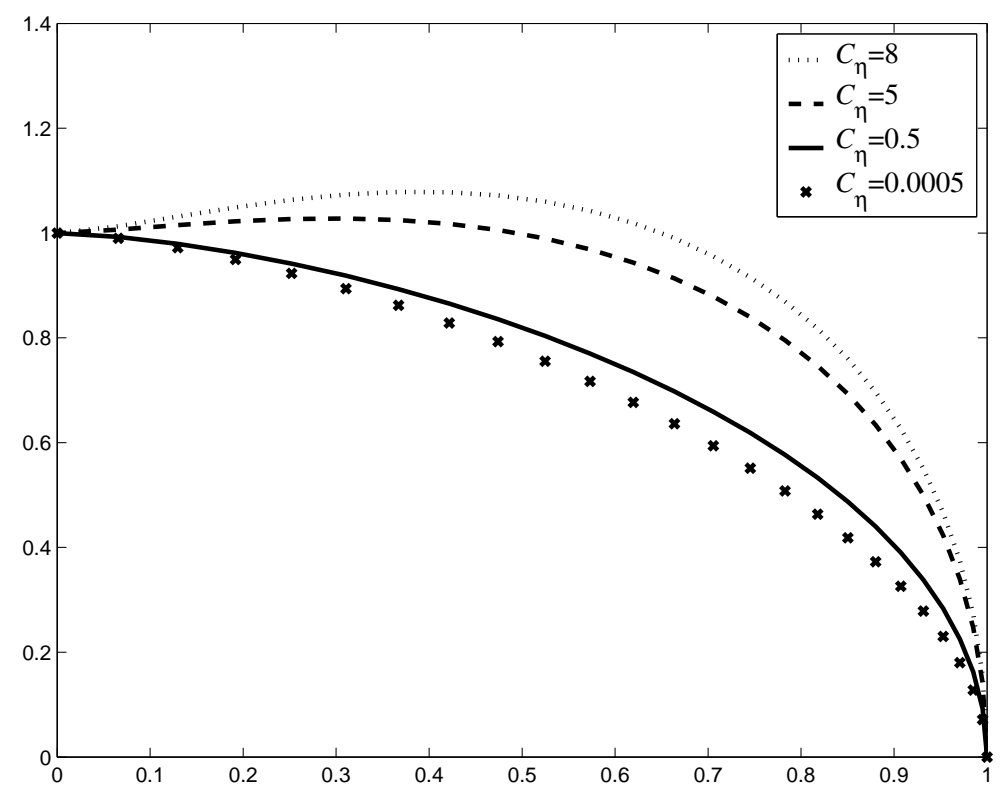

FIG. 3. Numerical solution of (4..3) (plots of $(\bar{h}(\bar{x})$ vs. $\bar{x})$ with $C_{\tau}=0.5$ for various values of $C_{\eta}$.

In figure 3, a fixed value of $C_{\tau}=0.5$ was used and solutions were computed for various values of $C_{\eta}$. We note that a similar conclusion to that highlighted in Section 2.6. for the paradigm problem applies, namely that as the value of $C_{\eta}$ increases, the solution becomes non-monotone with $\bar{h}(0+)>1$.

In figure 4 , a fixed value of $C_{\eta}=0.5$ was used and solutions were computed for various values of $C_{\tau}$. As $C_{\tau}$ increases, it is clear that the general shape of solutions changes. For larger values of $C_{\tau}$ it is not possible to obtain a numerical solution. The scheme does not converge, and all attempts to run with different parameters, proceed by continuation from "close by" solutions and weaken the convergence criteria fail. This suggests (but of course does not prove) that for these values of $C_{\tau}$ the problem has no solution, and it is thus possible that the full problem shares with the paradigm problem the characteristic that the solution becomes negative (and therefore unphysical) near to $\bar{x}=1$. 


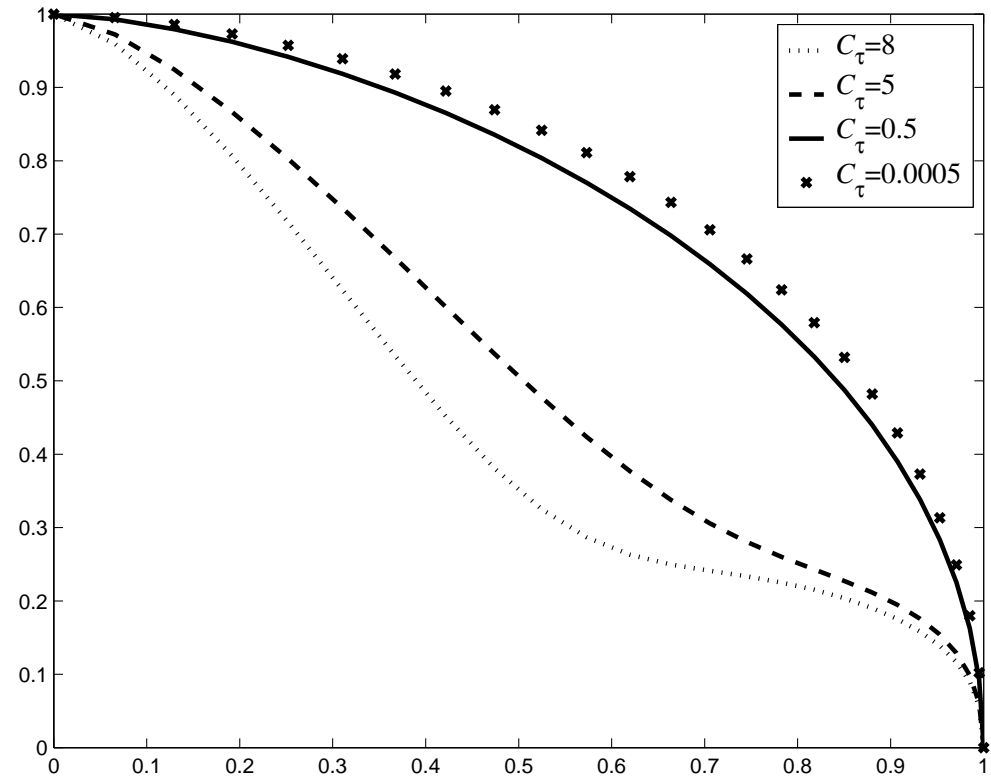

FIG. 4. Numerical solution of (4..3) (plots of $(\bar{h}(\bar{x})$ vs. $\bar{x})$ with $C_{\eta}=0.5$ for various values of $C_{\tau}$.

\subsection{Determination of $L$}

In this section we address the major objective of this study, namely the determination of of the dryout length $L$, and we also investigate how $L$ depends on the parameters $C_{\tau}$ and $C_{\eta} . L$ may be determined in an identical fashion to that employed in Section 2.6.. We find that

$$
L=-\frac{h_{0} \rho_{\infty} U_{\infty}^{2}}{\left(p_{\infty}-p_{g 0}\right) \pi} f_{0}^{1} \frac{\bar{h}_{\xi}(\xi)}{\xi} d \xi
$$

so that

$$
L=\frac{h_{0} \rho_{\infty} U_{\infty}^{2}}{\left(p_{\infty}-p_{g 0}\right)}\left(\frac{2}{\pi}+\frac{2}{\pi} f_{0}^{1} \frac{\sqrt{\xi(1-\xi)} F(\xi, \bar{h}(\xi))}{\xi} d \xi\right) .
$$

Values of $L$ were determined using (5..6) for the parameter values given in Appendix A, i.e. $\rho_{\infty}=$ $171 \mathrm{~kg} / \mathrm{m}^{3}, U_{\infty}=12 \mathrm{~m} / \mathrm{s}, H_{1}=1, p_{\infty}=200 \times 10^{5} \mathrm{~Pa}$ and $p_{g 0}=199.9 \times 10^{5} \mathrm{~Pa}$.

Table 1 below shows the relationship between the length to the dryout point $L$ and $C_{\tau}$ when the parameter $C_{\eta}$ is set equal to 1 . As might be expected from the results of Figure 4, an increase in $C_{\tau}$ results in a decrease in the thickness of the liquid film. As a consequence we would expect that an increase in the traction parameter $\bar{\tau}$ tends slowly but gradually to increase the liquid layer and hence the position of the dryout point. This tendency is confirmed by the results in Table 1.

Table 2 below shows the relationship between the length to the dryout point $L$ and $C_{\eta}$ when the parameter $C_{\tau}$ is set equal to 1 . It can be inferred from Table 2 that, as we would expect, for $C_{\eta} \ll 1$ the mass transfer $\bar{\eta}$ is small and dryout is delayed. By contrast, when $C_{\eta} \gg 1$ the mass transfer is so high that the liquid film cannot be established and dryout occurs immediately, leading to unphysical results. 


\begin{tabular}{|c|c|c|}
\hline$C_{\tau}$ & $C_{\eta}$ & $L$ \\
\hline 0.0001 & 1 & 1.242 \\
0.0005 & 1 & 1.242 \\
0.001 & 1 & 1.243 \\
0.005 & 1 & 1.244 \\
0.01 & 1 & 1.245 \\
0.1 & 1 & 1.275 \\
1 & 1 & 1.565 \\
2 & 1 & 1.884 \\
4 & 1 & 2.493 \\
10 & 1 & 4.069 \\
20 & 1 & 6.040 \\
30 & 1 & 7.671 \\
\hline
\end{tabular}

Table 1. Relationship between dryout length $L$ and parameter $C_{\tau}$ for a fixed value of $C_{\eta}$

\begin{tabular}{|c|c|c|}
\hline$C_{\tau}$ & $C_{\eta}$ & $L$ \\
\hline 1 & 0.0001 & 2.005 \\
1 & 0.0005 & 2.004 \\
1 & 0.001 & 2.004 \\
1 & 0.005 & 2.001 \\
1 & 0.01 & 1.996 \\
1 & 0.1 & 1.934 \\
1 & 1 & 1.565 \\
1 & 2 & 1.293 \\
1 & 4 & 0.882 \\
1 & 10 & 0.025 \\
1 & 20 & -1.027 \\
1 & 30 & -1.763 \\
\hline
\end{tabular}

Table 2. Relationship between dryout length $L$ and parameter $C_{\eta}$ for a fixed value of $C_{\tau}$

\section{Discussion and conclusions}

In this study we have proposed a model to determine the flow in a LMFBR boiler pipe. The key physical item of interest, namely the length to the dryout point, emerges from the model as a result of the flow calculation. The numerical results that are given in the previous section suggest that the values that are calculated for $L$ are physically reasonable. Interestingly, the results also exhibit remarkably similar trends as the results from a much more artificial (but much easier) paradigm problem.

The work that has been carried out also has some purely numerical implications. Equations such as (3..1) still present a numerical challenge. Though similar equations have been solved in a few previous studies (see, for example, (Fitt \& Wilmott, 1994) and (Fitt \& Pope, 2001)), the solution of such problems is by no means yet routine. Once again, the results of this study emphasise that careful asymptotic analysis and regularization are crucial for numerical success.

It is worth noting that we could have taken a completely different approach to the problem by treating it as a classical partial differential equation problem rather than by choosing, as we did, to formulate 
the free boundary problem in terms of an integro-differential equation. The advantage of our approach is that it allows the entire problem to be reduced to a single (albeit complicated) equation. Once this is solved, everything is known. Though it would clearly have been possible to use a purely numerical approach in solving the governing partial differential equations, the free boundary would have required careful treatment.

Of course, some major assumptions have been made in the model presented above. One might argue that, for practical purposes, it may be simpler just to measure the dryout point, but the safety constraints of an LMFBR make this a daunting experimental task. The model presented above not only provides key information about how changes in the upstream pipe flow might be expected to influence the position of the dryout point, but also describes how the key physical parameters in the problem influence the position of the dryout point. It also identifies the (relatively small) number of non-dimensional parameters that determine the flow.

It should also be noted that the model presented above is a steady version of an unsteady problem. One might wonder whether the full unsteady problem has stable solutions. Though this matter was partially addressed in (Mphaka, 2000)) it is clear that more work still needs to be carried out on the time-dependent problem.

Finally, it is worth pointing out that nuclear power is rapidly becoming fashionable again. Though the Douneray LMFBR was shut down in 1994 (along with the rest of the UK's LMFBR programme) it seems distinctly possible that, at some time in the not to distant future, Fast Breeder Reactors may once again be operating in the UK to provide a commercial source of power.

\section{References}

Anderssen, R.S., DE Hoog, F.R. \& LukAs, M.A. (EDS.) (1980) The Application and Numerical Solution of Integral Equations. Alphen aan den Rijn: Sijthoff and Noordhoff, 270pp.

AzBel, D. (1981) Two-phase flows in Chemical Engineering. Cambridge: Cambridge University Press, 331pp.

BANKHOFF, S.G. (1994) Significant questions in thin liquid film heat transfer, J. Heat Transfer, Trans. ASME, 116, 10-16.

Bennett, A.W., Hewitt, G.F., Kearsey, H.A., Keeys, R.K.F. \& Lacey, P.M.C. (1965) Flow visualization studies of boiling at high pressure, Proc. Inst. Mech. Eng., 180, 260-270.

Burelbach, J.P., BAnkhofF, S.G. \& DAVIS, S.H. (1988) Nonlinear stability of evaporating/condensing liquid films, J. Fluid Mech. 195, 463-494.

Collier, J.G. (1972) Convective Boiling and Condensation. London: McGraw Hill, 376pp.

Collier, J.G. \& HewitT, G.F. (1987) Introduction to Nuclear Power. Hemisphere Publishing Corporation, Berlin: Springer-Verlag, 231pp.

Collier, J.G. \& Thome, J.R. (1994) Convective Boiling and Condensation (3rd Edn.). Oxford: Clarendon Press, 596pp.

Cuminato, J.A., FitT, A.D. \& McKeE, S. (2007) A review of linear and nonlinear Cauchy singular integral and integro-differential equations arising in mechanics J. Integral Equations. Appl., 19, 163207. 
Dimitrakopoulos, P. \& Higdon, J.J.L. (1997) Displacement of fluid droplets from solid surfaces in low-Reynolds-number shear flows, J. Fluid Mech., 336, 351-378.

Fisher, S.A. \& PEARCE, D.L. (1993) An annular flow model for predicting liquid carryover into austenitic superheaters, Int. J. Multiphase Flow, 19, 295-307.

FitT, A.D. \& WilmotT, P. (1994) Slot film cooling - the effect of separation angle, Acta Mechanica, 103, 79-88.

FITT, A.D. \& Pope, M.P. (2001) The unsteady motion of two-dimensional flags with bending stiffness, J. Eng. Math., 40, 227-248.

Golberg, M.A. (1979) Solution Methods for Integral Equations: Theory and Applications. London: Plenum Press, 350pp.

Higuera, F.J. (1987) The hydrodynamic stability of an evaporating liquid, Phys. Fluids, 30, 679686.

Irvine, T.F. \& Hartnett, J.P. (EDS.) (1976) Steam and Air Tables in SI units. Washington: Hemisphere Pub. Corp., 127pp.

KING, A.C. \& TUCK, E.O. (1993) Thin liquid layers supported by steady air-flow surface traction, $J$. Fluid Mech., 251, 709-718.

Kirillov, P.L., Kashcheyev, V.M., Muranov, Yu. V. \& Yuriev, Yu.S. (1987) A twodimensional mathematical-model of annular-dispersed and dispersed flows I, Int. J. Heat Mass Transfer, 30, 791-800.

Kirillov, P.L., Kashcheyev, V.M., Muranov, Yu. V. \& Yuriev, Yu.S. (1987) A twodimensional mathematical-model of annular-dispersed and dispersed flows II, Int. J. Heat Mass Transfer, 30, 801-806.

KRENK, S. (1975) On quadrature formulas for singular integral equations of the first and second kind, Quart. Appl. Math., 33, 225-232.

Mphaka, M.J.S. (2000) Partial Singular Integro-differential Equation Models for Dryout in Boilers. Thesis (PhD). University of Southampton, 172pp.

MurRay. R.L. (1996) Nuclear Energy, (4th Edn.). Elsevier, 480pp.

MusKhelishVili, N.I. (1953) Singular Integral Equations. Groningen: Noordhoff, 447pp.

Prosperetti, A. \& Plesset, M.S. (1984) The stability of an evaporating liquid surface, Phys. Fluids, 27, 1590-1602.

Rubenstein, L.I. (1971) The Stefan problem; Transactions of Mathematical Monographs, vol. 27 New York: American Mathematical Society, 419pp.

Sadatomi, M., Kawaji, M., Lorencez, C.M. \& Chang, T. (1993) Prediction of liquid level distribution in horizontal gas-liquid stratified flows with interfacial level gradient, Int. J. Multiphase Flow, 19, 987-997. 
SAWAnt, P., Ishit, M. \& MORI, M. (2009) Prediction of amount of entrained droplets in vertical annular two-phase flow, Int. J. Heat Fluid Flow, 30, 715-728.

Schmidt, E. (ED.) (1969) Properties of Water and Steam in SI units. Berlin: Springer-Verlag, 205pp.

SPELT, P.D.M. (2006) Shear flow past two-dimensional droplets pinned or moving on an adhering channel wall at moderate Reynolds numbers: a numerical study, J. Fluid Mech., 561, 439-463.

ThwaItes, B. (1960) Incompressible Aerodymanics. Oxford: Oxford University Press, 636pp.

Van Dyke, M. (1975) Perturbation Methods in Fluid Mechanics. Stanford, CA.: Parabolic Press, 284pp.

Wallis, G.B. (1969) One-dimensional Two-phase Flow. New York: McGraw-Hill, 408pp.

Whalley, P.B. (1977) The calculation of dryout in a rod bundle, Int J. Multiphase Flow, 3, 501-515.

Whalley, P.B. (1987) Boiling, Condensation and Gas-liquid Flow. Oxford: Clarendon Press, 300pp.

Wilson, S.K., DAVID, S.H. \& BANKhofF, S.G. (1999) The unsteady expansion and contraction of a long two-dimensional vapour bubble between superheated or subcooled parallel plates, J. Fluid Mech., 391, 1-27. 


\section{Appendix A: Nomenclature and Boiler Tube Typical Values}

The values below apply to typical operating conditions in a LMFBR boiler and are taken from (Mphaka, 2000)-“Mp", (Irvine \& Hartnett, 1976)-“IH” and (Schmidt, 1969)-“Sc”. It is worth noting that, as far as parameter values are concerned, the literature is not unanimous and different sources may give different values.

$a \quad$ Typical tube radius $(\sim 7 \mathrm{~mm})(\mathrm{Mp})$

$h_{0} \quad$ Typical thickness of wall liquid layer $(\sim 0.1-1 \mathrm{~mm})(\mathrm{Mp})$

$L \quad$ Length to dryout point $(\sim 5 \mathrm{~m})(\mathrm{Mp})$

$L_{t} \quad$ Typical heated length of boiler pipe $(\sim 6.1 \mathrm{~m})(\mathrm{Mp})$

$g \quad$ acceleration due to gravity $\left(\sim 9.8 \mathrm{~m} / \mathrm{s}^{2}\right)$

$\varepsilon \quad$ Small parameter $h_{0} / L\left(\sim 10^{-4}\right)$

$c_{p} \quad$ Typical liquid specific heat $(\sim 15.646 \mathrm{~kJ} / \mathrm{kg} / \mathrm{K}$ at $180 \mathrm{bar}, 633 \mathrm{~K})(\mathrm{Sc})$

$k \quad$ Typical liquid thermal conductivity $(\sim 0.412 \mathrm{~W} / \mathrm{m} / \mathrm{K}$ at $200 \mathrm{bar}, 633 \mathrm{~K})(\mathrm{Sc})$

$\lambda \quad$ Typical latent heat of vapourisation of water $(\sim 607 \mathrm{~kJ} / \mathrm{kg}$ at $198 \mathrm{bar}, 638 \mathrm{~K})(\mathrm{IH})$

$q \quad$ Typical heat flux from liquid sodium $\left(\sim 595 \mathrm{~W} / \mathrm{m}^{2}\right)(\mathrm{Mp})$

$\mu \quad$ Typical dynamic viscosity of liquid $\left(\sim 6.68 \times 10^{-5} \mathrm{~N} \mathrm{sec} / \mathrm{m}^{2}\right.$ at $\left.200 \mathrm{bar}, 633 \mathrm{~K}\right)$ (Sc)

$\mu_{g} \quad$ Typical dynamic viscosity of vapour $\left(\sim 2.96 \times 10^{-5} \mathrm{~N} \mathrm{sec} / \mathrm{m}^{2}\right.$ at $\left.200 \mathrm{bar}, 633 \mathrm{~K}\right)(\mathrm{Sc})$

$\rho \quad$ Typical density of liquid ( $\sim 498 \mathrm{~kg} / \mathrm{m}^{3}$ at 198 bar, $\left.638 \mathrm{~K}\right)(\mathrm{IH})$

$\rho_{\infty} \quad$ Typical density of gas core flow upstream of dryout $\left(\sim 171 \mathrm{~kg} / \mathrm{m}^{3}\right.$ at $\left.200 \mathrm{bar}\right)(\mathrm{Sc})$

$\sigma \quad$ Surface tension $(\mathrm{N} / \mathrm{m})(\sim 0.002$ at $633 \mathrm{~K})(\mathrm{Sc})$

$\dot{M} \quad$ Dimensional mass flow from liquid to gas core $\left(\mathrm{kg} / \mathrm{s} / \mathrm{m}^{2}\right)$

$\dot{m} \quad$ Non-dimensional mass flow from liquid to gas

$p_{\infty} \quad$ Typical pressure in the gas core far upstream of dryout $(\sim 200$ bar) $(\mathrm{Mp})$

$U_{\infty} \quad$ Typical gas core velocity $(\sim 12 \mathrm{~m} / \mathrm{s})(\mathrm{Mp})$

$U \quad$ Typical liquid velocity $(\sim 0.01 \mathrm{~m} / \mathrm{s})(\mathrm{Mp})$

$T_{s} \quad$ Typical saturation temperature $(\sim 638.86 \mathrm{~K}$ at $200 \mathrm{bar})(\mathrm{IH})$

$T_{w} \quad$ Typical wall temperature $(\sim 640 \mathrm{~K})(\mathrm{Mp})$ 